\title{
'How the introduction of a gastrostomy algorithm influenced the incidence of peristomal wound infection'
}

\author{
S. Skerratt, J. Anderson, H. Lawrence, L. Russell and J. Woodward \\ Addenbrookes Hospital,Cambridge, UK
}

Percutaneous endoscopic gastrostomy (PEG) is the method of choice for long-term artificial enteral feeding. At Addenbrooke's Hospital, it is now standard nursing practice to use a gastrostomy pre- and post-care algorithm in the prevention of peristomal wound infection. It has been reported that 5-30\% of PEG patients will experience a peristomal wound infection ${ }^{(1)}$ and commonly isolated organisms are Streptococcus, Staphylococcus, Coliforms and anerobes. Peristomal wound infections are the most common complication of PEG, despite prophylactic antibiotics. The aim of this review was to establish whether the algorithm is influential in reducing infection rates during the immediate 14-day post procedure follow-up.

We analysed a total of 160 patients undergoing gastrostomy and jejeunostomy placements between August 2007 and April 2009. They were identified from the endoscopy database and electronic medical records. Insertion dates were confirmed from data collected by the Nutrition Nurse Specialists and reviewed. As stated in the algorithm all patients were to be given IV Gentamicin together with a body wash of Octenisan prior to insertion. On day 1 post procedure, the peristomal site dressing was removed, the site cleaned with normal saline and Mupirocin 2\% applied topically. No dressing was required. On days 3-7 the site was cleaned with Octenisan body wash and Mupirocin $2 \%$ reapplied to the peristomal site. From day 7 onwards the peristomal site was able to be cleaned daily using warm soapy water and there was no further requirement for Mupirocin. Instructions were given not to immerse the site in water until day 7 . At day 7 once the gastrostomy/jejeunostomy tract was healed, the flange could be loosened if so desired. Depending on the type of gastrostomy/ jejeunostomy in situ, insertion and rotation or balloon checks could be commenced. The peristomal site was evaluated on days 3,7 and 14 following insertion by follow up visits to the wards, outpatient clinics or telephone calls to the usual place of residence. Erythema and Exudate were scored on a scale from 0 to 4; induration was scored on a scale of 0-3. A maximum combined score of more than 3 could be suggestive of an infection and further investigation would be required ${ }^{(2,3)}$.

Between August 2007 and March 2008, 49 patients had gastrostomy/jejeunostomy placements. Nine out of 49 (18\%) patients had signs (score >3) suggestive of an infection. In April 2008, the algorithm was introduced to all wards and the community together with changes in endoscopy practices. This resulted in post-gastrostomy procedure infection rates falling from $18 \%$ to $0.9 \%$ (1 out of 111 ) patients that were seen from April 2008 to April 2009.

In conclusion, this study demonstrates that carefully monitored surveillance and adherence to routine principles of wound care together with the use of this algorithm is an effective way to deliver gastrostomy care, minimise infection and long-term complications.

1. Jonas SK, Neimark S \& Panwalker AP (1985) Am J Gastroenterol 6, 438-441.

2. Ahmad I, Mouncher A, Abdoolah A et al. (2003) Aliment Pharmacol Ther 18, 209-215.

3. Naresh K, Jain MD et al. (1987) Ann Intern Med 107, 824-828. 